\title{
Automatic Trimming Algorithm and Process Testing of Precise Carbon Film Potentiometer
}

\author{
Yuefeng $\mathrm{Li}^{1,2, a}$, Huifeng Wang ${ }^{1}$, Guanglin Wang ${ }^{1}$ and Li Sun ${ }^{2}$ \\ ${ }^{1}$ School of Mechanical Engineering, Harbin Institute of Technology, Harbin, China, 150001 \\ ${ }^{2}$ School of Electrical Engineering \& Automation, Harbin Institute of Technology, Harbin, China, \\ 150001 \\ a lilu1001@163.com
}

Keywords: Precise carbon film potentiometer, Automatic linearity trimming machine, Linearity trimming algorithm, Error compensation.

\begin{abstract}
The precise carbon film potentiometer (PCFP) is widely used in the servo system. For gaining the high precision and production efficiency, a new trimming algorithm has been proposed and a new error compensation technology has been used in this study. By using the newly developed automatic linearity trimming machine ALTM, the precision and efficiency have been increased significantly, which can solve the contradiction between supply and demand of PCFP. The test results show that the designed ALTM is able to reach the target.
\end{abstract}

\section{Introduction}

Precision carbon film potentiometer (PCFP) is widely used as the position feedback component belonging to servomechanism in the area of aviation and aerospace field. It is a kind of synthetic carbon film potentiometer formed by spraying the resistance liquid, which contains graphite, carbon black and resin etc., on a piece of insulation framework and then stoving, polymerizing[1-2]. It can be classified into two categories by its shape-linear type and circle type. The advantages of this kind of potentiometer are high resolution, wide range of resistance, good abrasion resistance, simple process and low cost. There are many indexes in evaluating a potentiometer, the most significant of which is the nonlinear precision, which is the deviation between actual output and theory output.

There are two key techniques in producing PCFP. The first one is the configuration of synthetic carbon film size and coating process. This is the first step of producing PCFP; however, it is hardly to produce high precision potentiometer, as mentioned above, through coating process alone[3]. The second one is the trimming technology. Due to uncertainty of the coating process, the size and cross sectional area of the coat is not uniform, making the nonlinear error not meet the request. Usually, detection and trimming, before that the error is around $2 \%$, are required to control the nonlinear error in a certain range which is commonly below $0.5 \%$.

The traditional trimming method, which is low efficiency, nearly fifty minutes per piece, and high labor intensity, in keeping the linearity is trimming manually using bridge and grinding wheel and require professional operator, which can hardly meet volume production. Therefore, an automatic linearity trimming machine (ALTM), both enhancing precision and increasing trimming speed, is made under the control of industrial computer. The ALTM consists of mechanical part and control circuit[4-6] and can raise the precision to $0.1 \% \sim 0.2 \%$ and reduce the processing time to around four minutes per piece.

\section{Linearity Trimming Algorithm}

Online feedback trimming method is proposed in this paper. Sensors are used during the process of PCFP to collect parameters online. According to the differences between these parameters and the theory ones, ALTM could carve the PCFP into the target value and meet the nonlinear requirement. 
Main Technical Index of PCFP. There are many technical indexes in evaluating a PCFP, such as the length of the carbon film, the all-in resistance, the left and right resistance, the unistor, the bring-in resistance and the nonlinear error. The most significant one is the nonlinear error.

In the Fig. 1, the ideal result is that the relationship between $\mathrm{Xi}$ and $\mathrm{Vi}$ is linear, however there is relative error, the maximum of which is called maximum nonlinear error, between the actual and theoretical voltage output as shown in the Fig. 2.
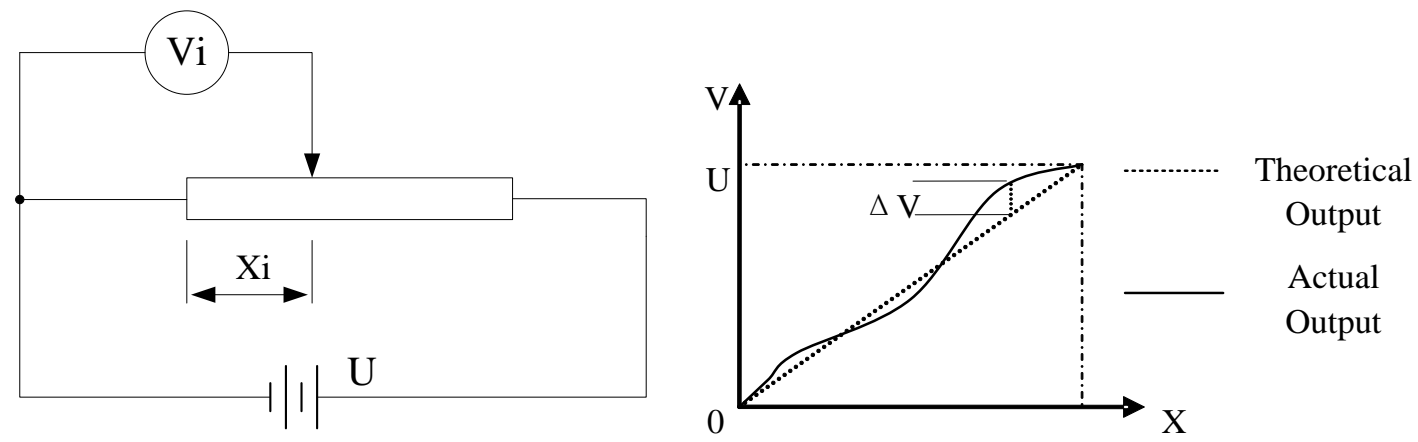

Fig.1 Non-linear error measurement circuit Fig.2 Potentiometer voltage output

$$
\delta=\frac{\Delta V_{\max }}{U} \times 100 \%
$$

The nonlinear error of each position is $\delta_{i}$,

$$
\delta_{i}=\frac{v_{i}-u_{i}}{U}=\frac{v_{i}-\frac{x_{i}}{L} \times U}{U}=\left(\frac{v_{i}}{U}-\frac{x_{i}}{L}\right) \times 100 \%
$$

Basic Principle of Automatic Trimming Algorithm. The target is to wipe out the redundant resistance of each position and gain the linear output curve, however how much carbon films should the ALTM wipe out follows the formula below:

$$
\Delta r_{i}=r_{i}^{\prime}-r_{i}
$$

This shows that it is significant to get the accurate resistance of each position, and there are two ways in measuring the resistance value - one is resistance method and the other is voltage method as shown in the Fig. 3.

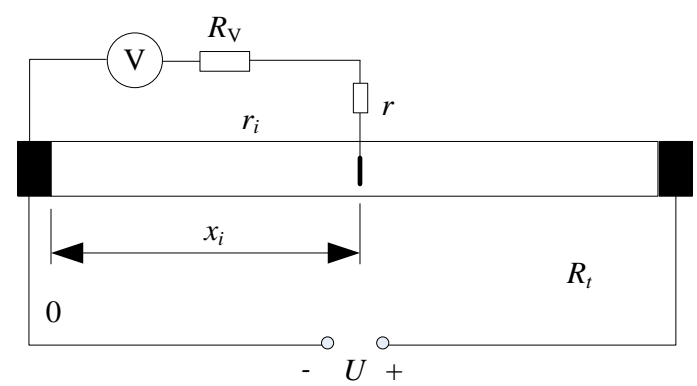

Fig.3 View of partial voltage to measure resistance

Measure the resistance of each position with electric brush directly. (resistance method) When the multimeter is on its resistance stall, $R v$ is very small while $r=10 \sim 30 \Omega$, and this will bring in nonlinear error. Suppose $R t=3000 \Omega$, then

$$
\begin{gathered}
\delta_{1}=\frac{10 \Omega}{3000 \Omega} \times 100 \%=0.333 \% \\
\delta_{2}=\frac{30 \Omega}{3000 \Omega} \times 100 \%=1.0 \%
\end{gathered}
$$


Apparently, it does not conform to the requirements. Calculate the resistance with the voltage of that position. (voltage method). When the multimeter is on its voltage stall, $R v$ is large enough which is up to $100 \mathrm{M} \Omega$ when the range is below $100 \mathrm{~V}$ according to the instruction. Then the calculate resistance with partial voltage of position $\mathrm{Xi}$ is

$$
r_{\mathrm{c}}=\frac{\left(R_{\mathrm{V}}+r\right) \times r_{i}}{R_{\mathrm{V}}+r+r_{i}}
$$

Suppose $\mathrm{ri}=2000 \Omega, \mathrm{r}=20 \Omega$, then

$$
\delta=\frac{r_{i}-r_{c}}{R_{t}}=\frac{0.37 \Omega}{3000 \Omega} \times 100 \%=0.012 \%
$$

Apparently, the second method is better, then

$$
\Delta r_{i}=r_{i}^{\prime}-r_{i}=\frac{X_{i}}{L} \times R_{t}-\frac{R_{t}}{U} \times V_{i}
$$

It is not a proper way to acquire $\Delta r_{i}$ with electric brush, not only which will bring in contact resistance but the measured results will be affected by the vibration during processing as well. A new theory, which is the basic theory of ALTM, called all-in resistance feedback theory, electric brush excluded, is proposed, that the resistance changes in each position could be reflect in the all-in resistance and their value are equal.

\section{Linearity Trimming Algorithm}

The ALTM can automatically machining the PCFP to meet the nonlinear error requirement and other indexes. It is controlled by industrial machine and contains mechanical part and electrical part as shown in the Fig. 4.

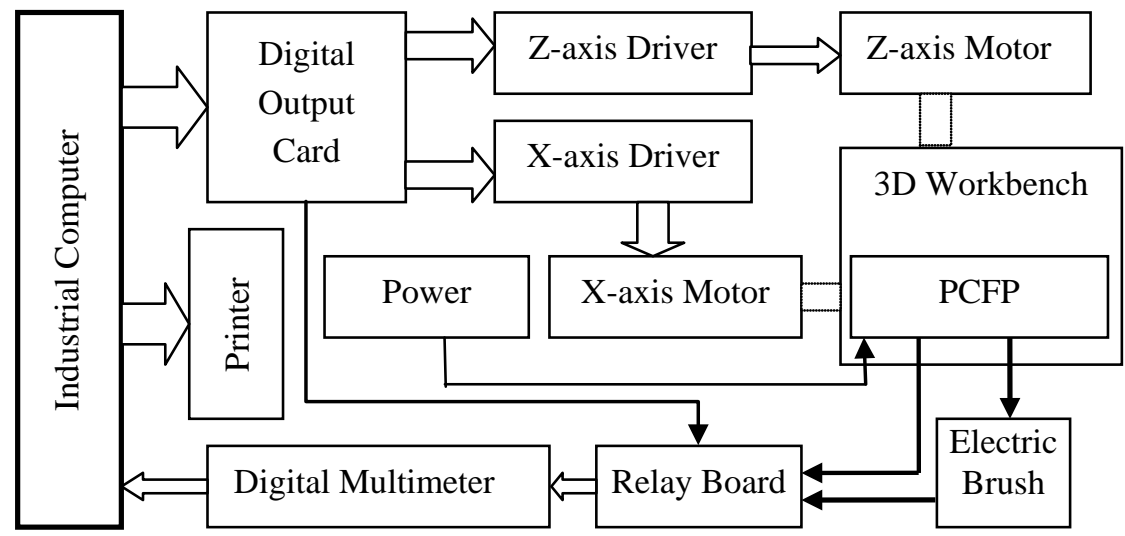

Fig.4 Sketch map of ALTM hardware

The mechanical part consists of numerical control three-dimensional workbench (3D NC workbench), electric brush organ and clamping apparatus. The 3D NC workbench control three axises (X, Y, Z), which consist of stepping motor, shaft coupling, screw pair and one pair of lead rail, through digital output card and NC program. Two position switches are installed at the extreme position of each axis to prevent the workbench overrun. The drill is controlled by a frequency converter which the maximum frequency is $400 \mathrm{~Hz}$. The electric brush organ plays an important role in measuring the resistance, and the electric brush is the only facility that touches the carbon film directly. Designing the brush organ, many factors listed below are considered. 


\section{Test results of trimming PFCP with ALTM}

Table 1 shows the changes of main technical parameters after trimming.

Table 1 PFCP-01 main parameters comparison before \& after trimming

\begin{tabular}{ccccc}
\hline $\begin{array}{c}\text { Param } \\
\text { eter }\end{array}$ & $\begin{array}{c}\text { Left } \\
\text { resistance( }\end{array}$ & $\begin{array}{c}\text { Right } \\
\text { resistance( }\end{array}$ & $\begin{array}{c}\text { All-in } \\
\text { resistance( })\end{array}$ & $\begin{array}{c}\text { Nonlinear } \\
\text { error }\end{array}$ \\
\hline $\begin{array}{c}\text { Raw } \\
\text { Proces }\end{array}$ & 1611.6 & 1616.2 & 3129.8 & $0.93 \%$ \\
sed & 1716.2 & 1715.4 & 3350.8 & $0.19 \%$ \\
\hline
\end{tabular}

Four other PFCP are dilled by this ALTM to test the system and Table 2 shows the differences between raw PFCP and processed PFCP in key indexes.

Table 2 Five PFCPs’ parameters comparison before \& after trimming

\begin{tabular}{|c|c|c|c|c|}
\hline \multirow[t]{3}{*}{ PFCP } & \multicolumn{2}{|c|}{ Before trimming } & \multicolumn{2}{|c|}{ After trimming } \\
\hline & Unistor & Nonlinear & Unistor & Nonlinear \\
\hline & ) & error & ( & error \\
\hline PFCP-01 & 4.6 & $0.93 \%$ & 0.8 & $0.19 \%$ \\
\hline PFCP-02 & 5.2 & $0.82 \%$ & 1.1 & $0.17 \%$ \\
\hline PFCP-03 & 4.8 & $1.01 \%$ & 1.2 & $0.20 \%$ \\
\hline PFCP-04 & 5.1 & $0.85 \%$ & 0.9 & $0.18 \%$ \\
\hline PFCP-05 & 4.9 & $0.91 \%$ & 1.1 & $0.19 \%$ \\
\hline
\end{tabular}

The nonlinear error, which is the most significant index in evaluating a PFCP, and unistor have been improved drastically and reach the designing target.

\section{Conclusions}

In this paper, an automatic trimming algorithm is proposed and the testing system ALTM is designed, based on which a process testing is made whose results proves the accuracy of the algorithm. The ALTM could drill two kinds of PFCP, center tap and non-center tape, with different length. The precision and the efficiency have been improved drastically. The ALTM will play an important role in quantity production of PFCP.

\section{Acknowledgement}

This material is based upon work supported by the research and innovation fund of Harbin Institute of Technology (Grant Number: HIT.NSRIF.2015 053).

\section{References}

[1] Bewi loguak, Cooper. Effect of targetmaterial on depositi on and properties of metal-containing DLC (Me-DLC) coatings, Surface and Coatings Technology. 127 (2000) 224-232.

[2] A.F. Ismail, David L I B, A Review on the Latest Development of Carbon Membranes for Gas Separation. J. Membr. Sci. 193 (2001) 1-18.

[3] W.L. Zhou, M. Yoshino, Kina H, et al. Preparation and Gas Permeation Properties of Carbon Molecular Sieve Membrane Based on Sulfonated Phenolic Resin, J. Membr. Sci. 217 (2003) 55-67.

[4] S. Schofield, P. Wright. Open Architecture Controllers for Machine Tools, Design Principles, Transaction of the ASME, 120 (1998) 417-424.

[5] J.W. Jeon, Y.Y. Ha. A Generalized Approach for the Acceleration and Deceleration of Industrial Robotics and CNC Machine Tools. IEEE Transaction On Industrial Electronics. 47 (2000) 115-120

[6] M. Hideo, S. Chihiro. Japanese PC-based control systems for manufacturing equipment. International Journal of Japan Society Precision Engineering. 30 (1996) 204-209. 